\section{The ESR Studies of Solubilised Nitroxide Spin Probes in Cetyltrimethylammonium Bromide Micelles}

\author{
Hirofumi Okabayashi*, Koji Kihara, \\ and Masataka Okuyama \\ Department of Industrial Chemistry, \\ Nagoya Institute of Technology, \\ Gokiso, Showa-ku, Nagoya 466, Japan \\ (Z. Naturforsch. 32 a, 1571-1573 [1977]; \\ received November 12, 1977)
}

The rotational correlation times of two long-chain nitroxide spin probes (5-PASL and 1-LASL) were measured in the vicinity of the second critical micelle concentration of cetyltrimethylammonium bromide (CTAB) solutions. The radical part of 5-PASL is situated at the $\delta$-carbon and that of 1-LASL in the polar head.

In the 5-PASL-solubilized CTAB solutions a transition of the correlation time due to a sphere-rod transition seems to occur, while in the 1-LASL-solubilized CTAB solutions no such transition occurs. This reveals that the hydrophobic part around the $\delta$-carbon of the probe becomes more rigid as a result of the micellar shape-variation while the hydrophilic part of 1-LASL is not so much restricted by the shape variation.

Nitroxide free-radical probes can be used for investigations of the structure of micelles, and it has been shown that for probe-solubilized micelle systems a sharp transition of the rotational correlation time occurs at the first critical micelle concentration (CMC) ${ }^{1-4}$. This transition has been ascribed to the fact that the tumbling rate of the probe molecule is slowed down because of interaction with the micelle.

In the present work, two long-chain nitroxideradical probes were used to investigate the change of the rotational correlation times of the probes due to the sphere-rod transition of the CTAB micelles.

\section{Experimental}

Cetyltrimethylammonium bromide (CTAB) and cetyltrimethylammonium chloride (CTAC) were purchased from Nakarai Chemicals LTD (Japan) and were purified by recrystallisation from acetonethanol. The N-oxyl-4', $4^{\prime}$-dimethyloxazolidine derivative of 5-ketopalmitic acid (5-PASL) and the 2,2-6,6-tetramethyl-4-piperidinol-1-oxyl ester of lauric acid (1-LASL) were synthesized according to

Requests for reprints should be sent to Dr. H. Okabayashi, Department of Industrial Chemistry, Nagoya Institute of Technology, Gokiso, Showa-ku, Nagoya 466, Japan. the procedures of references ${ }^{2,5}$. These spin-labeled substances were purified by column chromatography.

$$
\mathrm{CH}_{3}-\left(\mathrm{CH}_{2}\right)_{10}-\mathrm{C}-\left(\mathrm{CH}_{2}\right)_{3}-\mathrm{COOH}
$$

5-PASL

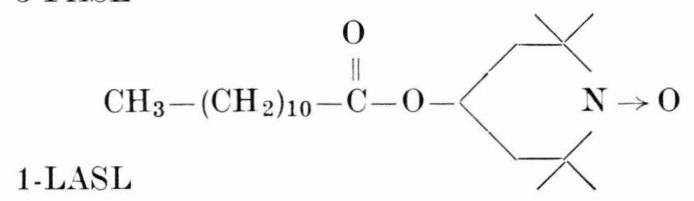

The ESR spectra were taken with a JEOL-model JES-ME spectrometer at room temperature (22 $\left.\pm 2{ }^{\circ} \mathrm{C}\right)$.

\section{Results and Discussion}

The tumbling rate of a nitroxide radical is usually expressed by a rotational correlation time $\left(\tau_{\mathrm{c}}\right)$, which has been related to the ESR spectral line-widths ${ }^{6}$. For the narrow-line ESR-spectra of the radical, two estimates $\left(\tau_{\mathrm{c}}\right.$ and $\left.\tau_{\mathrm{c}}{ }^{\prime}\right)$ of the correlation time in nanoseconds can be evaluated by the following equations $1,6-8$ :

$$
\begin{aligned}
& \tau_{\mathrm{c}}=0.65 W_{0}\left[\left(h_{0} / h_{1}\right)^{1 / 2}+\left(h_{0} / h_{-1}\right)^{1 / 2}-2\right], \\
& \tau_{\mathrm{c}}{ }^{\prime}=-2211 W_{0} / H_{0}\left[\left(h_{0} / h_{1}\right)^{1 / 2}-\left(h_{0} / h_{-1}\right)^{1 / 2}\right] .
\end{aligned}
$$

$W_{0}$ is the line-width of the central peak and $H_{0}$ the applied magnetic field, both measured in Gauss. $h_{0,1,-1}$ is height of the middle, low, and high field lines, respectively. $\tau_{\mathrm{c}}$ should be equal to $\tau_{\mathbf{c}}{ }^{\prime}$ for isotropic motion of the probe. However, it is well known that the above two equations often give different values. The difference $\left(\Delta \tau_{\mathrm{c}}\right)$ between $\tau_{\mathrm{c}}$ and $\tau_{\mathbf{c}}{ }^{\prime}$ has been regarded as a measure of the anisotropy of the motion 4,9 .

In the present study, the rotational correlation times of 5-PASL and 1-LASL incorporated into CTBA micelles were measured in the vicinity of the second CMC. In calculations of the correlation times, the effects of chemical exchange between monomer and micelle were neglected, because the micelle concentration is very high in this study. Furthermore, since in surfactant solutions the reduction of rotational motion of a nitroxide probe is the predominant reason for the broadening of the ESR spectrum, the effects of hopping between polar and non-polar sites were also neglected. 
In saturated aqueous solutions of 5-PASL, the ESR spectrum consists of three lines of nearly equal line-width. When, however, 5-PASL is solubilised in CTAB micelles, the widths of the three hyperfine lines become unequal.

Table I shows the $\tau_{\mathrm{c}}$ and $\tau_{\mathrm{c}}{ }^{\prime}$ values of 5-PASL and 1-LASL in organic solvents and CTAB micelles. In the 5-PASL-CTAB micelles, $\Delta \tau_{\mathrm{c}}$ is much larger than in organic solvents. This reveals that the tumbling motion of 5-PASL is slowed down as a result of incorporation into the micelles.

Table I. Rotational correlation times for 5-PASL and 1-LASL in organic solvents and in CTAB micelles $\left(\times 10^{-11} \mathrm{~s}\right)$.

\begin{tabular}{|c|c|c|c|c|c|c|}
\hline & \multicolumn{3}{|c|}{ 5-PASL } & \multicolumn{3}{|c|}{ 1-LASL } \\
\hline & $\tau_{\mathrm{c}}$ & $\tau_{\mathrm{c}}^{\prime}$ & $\Delta \tau_{\mathrm{c}}$ & $\tau_{\mathrm{c}}$ & $\tau_{\mathrm{c}}^{\prime}$ & $\Delta \tau_{\mathrm{c}}$ \\
\hline water & 23.6 & 18.0 & 5.6 & - & - & - \\
\hline ethyl alcohol & 17.8 & 31.7 & 13.9 & 12.3 & 3.4 & 8.9 \\
\hline hexyl alcohol & 56.0 & 54.8 & 1.2 & 25.1 & 13.6 & 11.5 \\
\hline benzene & 1.0 & 9.4 & 8.4 & 2.3 & 3.5 & 1.2 \\
\hline hexadecane & 33.1 & 31.3 & 1.8 & 7.1 & 10.4 & 3.3 \\
\hline $\begin{array}{l}\text { CTAB solution } \\
\left(120 \mathrm{mg} / \mathrm{cm}^{3}\right)\end{array}$ & 290.0 & 174.0 & 126.0 & 87.6 & 96.1 & 8.5 \\
\hline
\end{tabular}

In the 5-PASL-saturated aqueous solution, $\Delta \tau_{\mathrm{c}}$ does not differ appreciably from the values in organic solvents. Accordingly, the 5-PASL-molecules are monomeric in the aqueous solution and the molecular motion is almost isotropic. $\Delta \tau_{\mathrm{c}}$ of 5-PASL in the CTAB micelles is much larger than in organic solvents. This shows that the molecular motion of 5-PASL becomes anisotropic in the CTAB micelles.

In the case of the 1-LASL-CTAB micelles, $\tau_{\mathrm{c}}$ is evidently small compared to that in the 5-PASLCTAB micelles. Moreover, $\Delta \tau_{\mathrm{c}}$ of 1 -LASL in the CTAB micelles is very close to the values in organic solvents. This shows that in the CTAB micelles the radical part of 1-LASL is less hindered than the radical part of 5-PASL, and the molecular motion is not anisotropic.

The rotational correlation time of 5-PASL is ploted as a function of the CTAB concentration in Figure 1. It is noteworthy that a transition of $\tau_{\mathrm{c}}$ occurs in the range of $90-120 \mathrm{mg} / \mathrm{cm}^{3}$. This range closely corresponds to the second CMC 10 . On the other hand, when 5-PASL is solubilised in CTAC micelles, no such transition occurs; in this case there is no second CMC. Thus the transition should be ascribed to a change of the micellar shape.
Figure 1 also shows $\tau_{\mathrm{c}}$ of 1-LASL as a function of the CTAB concentration. For this probe, there is markedly little dependence of $\tau_{\mathrm{c}}$ on the CTAB concentration. Thus, it may be concluded that the rigidity of the polar head of the CTAB micelle does not change markedly with the micellar shape.

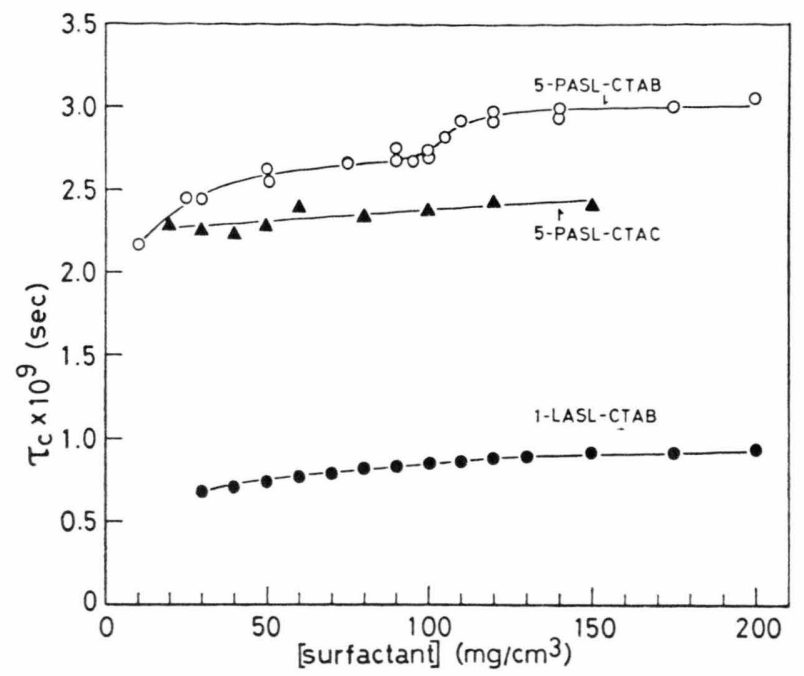

Fig. 1. Rotational correlation times of 5-PASL and 1-LASL in aqueous solutions vs. surfactant concentrations (room temperature: $22 \pm 2{ }^{\circ} \mathrm{C}$ ).

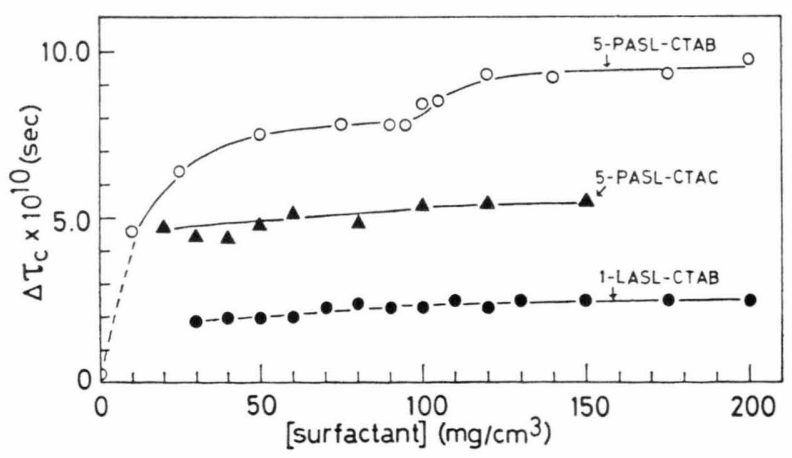

Fig. 2. $\Delta \tau_{\mathrm{c}}$ values of 5-PASL and 1-LASL in aqueous solutions vs. surfactant concentrations.

Figure 2 shows the $\Delta \tau_{\mathrm{c}}$ values of 5 -PASL and 1-LASL as a function of the surfactant concentration. For the 5-PASL-CTAB solutions, at ca. $100 \mathrm{mg} / \mathrm{cm}^{3}$ the transition of $\Delta \tau_{\mathrm{c}}$ also seems to occur, while in the 5-PASL-CTAC system no transition is found. This observation shows that the molecular motion of 5-PASL becomes more anisotropic because ofthe sphere rod transition. When 1-LASL is solubilised in the CTAB micelles, 
$\Delta \tau_{\mathrm{c}}$ remains unchanged. Accordingly, we can concluded that in the polar part of the CTAB micelles the intramolecular rotational motion of the radical part is not so hindered in spite of the micellar shape-variation, in contrast to the hydrocarbon part of the micelles.
1 A. S. Waggoner, O. H. Griffith, and C. R. Christensen, Proc. Nat. Acad. Sci. U.S. 57, 1198 [1967].

2 A. S. Waggoner, A. D. Keith, and O. H. Griffith, J. Phys. Chem. 72, 4129 [1968].

3 S. Ohnishi, T. J. R. Cyr, and H. Fukushima, Bull. Chem. Soc. Japan. 43, 673 [1970].

4 J. R. Ernandes, S. Schreier, and H. Chaimovich, Chem. Phys. Lipids 16, 19 [1976].

5 W. L. Hubbell and M. H. McConnell, J. Amer. Chem. Soc. 93, 313 [1971].
6 D. Kivelson, J. Chem. Phys. 27, 1087 [1957].

7 M. B. McBride, J. Phys. Chem. 80, 196 [1976].

8 F. Sachs and R. Latorre, Biophys. J. 14, 316 [1974].

9 B. Cannon, C. F. Polnaszek, K. W. Butler, L. E. Goran Eriksson, and I. C. P. Smith, Arch. Biochem. Biophys. 167, 505 [1975].

10 P. Ekwall, L. Mandell, and P. Solyom, J. Colloid Interf. Sci. 35, 519 [1971]. 
Nachdruck - auch auszugsweise - nur mit schriftlicher Genehmigung des Verlags

Verantwortlich für den Inhalt: A. KLEMM

Satz und Druck: Konrad Triltsch, Würzburg 